\title{
Effects of 5HT1A Activation on Gating Profile Following 5HT Depletion in Rats Lacking Social Attachment Since Weanling
}

\author{
Yueh-Ming Tai ${ }^{*}$, Chih-Yuan $\mathrm{Ko}^{2,3^{*}}$, Chen-Cheng Lin ${ }^{4}$, Yu-Yue Wan ${ }^{4}$, Jing-Yi Chung ${ }^{4}$, and Yia-Ping Liu ${ }^{4,5 凶}$ \\ ${ }^{1}$ Department of Psychiatry, Beitou Branch, Tri-Service General Hospital, National Defense Medical Center, Taipei, Taiwan \\ ${ }^{2}$ Respiratory Medicine Center of Fujian Province, Quanzhou, China \\ ${ }^{3}$ Department of Respiratory and Critical Care Medicine, the Second Affiliated Hospital of Fujian Medical University, Quanzhou, China \\ ${ }^{4}$ Laboratory of Cognitive Neuroscience, Department of Physiology and Biophysics, National Defense Medical Center, Taipei, Taiwan \\ ${ }^{5}$ Department of Psychiatry, Tri-Service General Hospital, Taipei, Taiwan
}

\begin{abstract}
Objective Central 5-HT1A receptor is involved in the modulation of sensorimotor gating function. However, its precise role is not clearly defined in developmentally social deprived (isolation rearing, IR) rats featured with impaired sensorimotor gating ability. We therefore aimed to examine the effects of 5HT1A activation on acoustic startle response (ASR) and prepulse inhibition (PPI) in IR rats in a condition of compromised presynaptic 5-HT functions.
\end{abstract}

Methods Social control (SOC) and IR rats received an intracerebraoventricular (ICV) injection of 5-HT depletor, 5,7-DHT. Seven days later rats entered a protocol of 8-OH-DPAT, a 5-HT1A agonist, in which locomotor activity, ASR and PPI and their tissue levels of 5-HT were measured.

Results Our results found that both IR and 5,7-DHT decreased the tissue concentration of 5-HT. IR-induced hyperactivity and gating impairment were unaffected by 5-HT depletion. 8-OH-DPAT strengthened the ASR in IR but not SOC rats and the drug-reduced PPI could be adjusted by 5,7-DHT pretreatment. 8-OH-DPAT at $100 \mu \mathrm{g} / \mathrm{kg}$ enhanced PPI in 5-HT-depleted SOC rats. However for IR rats, 8-OH-DPAT strengthened PPI in sham rats but downgraded it in depletion condition.

Conclusion The integrity of central 5-HT system is important to 5-HT1A-modulated sensorimotor gating in isolation-reared rats.

Psychiatry Investig 2018;15(2):193-199

Key Words 5HT1A receptors, Isolation-rearing, Prepulse inhibition, Serotonin.

\section{INTRODUCTION}

The precise role of central serotonergic system in acoustic startle response (ASR) and prepulse inhibition (PPI) is not clearly defined. 8-OH-DPAT, a 5-HT1A receptor agonist, has been used to investigate this topic in rat models as it was reported to increase the magnitude of the startle response ${ }^{1}$ and decrease the PPI. ${ }^{2}$ Sipes and Geyer later confirmed the central involvement of 5-HT1A receptor in gating function by demonstrating that the reduction in PPI produced by intra-raphe

Received: December 18, 2016 Revised: February 6, 2017

Accepted: May 14, 2017 Available online: November 7, 2017

$\triangle$ Correspondence: Yia-Ping Liu, MD, PhD

Department of Physiology and Biophysics, National Defense Medical Center, No.161, Section 6, Min-Chuan East Road, Taipei 114, Taiwan, Republic of China Tel: +886 287923100 ext18614, Fax: +886 287923153

E-mail: yiaping@ms75.hinet.net

*These authors contributed equally to this work.

(c) This is an Open Access article distributed under the terms of the Creative Commons Attribution Non-Commercial License (http://creativecommons.org/licenses/by$\mathrm{nc} / 4.0$ ) which permits unrestricted non-commercial use, distribution, and reproduction in any medium, provided the original work is properly cited. microinjections of 8-OH-DPAT was prevented by a systemic injection of a selective 5-HT1A antagonist, (+) WAY 100135. ${ }^{3}$ These findings suggest that rats with enhanced 5-HT1A activity are inferior to execute sensorimotor gating function when encountering an acoustic startle stimulus.

Since the presynaptic 5-HT1A receptor serves as a somatodendritic autoreceptor, effects through this receptor are highly relevant to the presynaptic or releasing performance of $5-\mathrm{HT}^{4}{ }^{4}$ Study with 5-HT depletor, 5,7-dihydroxytryptoamine (5,7DHT) found that the effect of 8-OH-DPAT can be potentiated in 5-HT-depleted rats, ${ }^{5}$ with or without an impairment of baseline PPI (i.e., by 5,7-DHT per se)., ${ }^{5,6}$ This is particularly interesting because the 5,7-DHT lesioning, as it diminishes the presynaptic 5-HT function, is expected to ameliorate the PPI deficit induced by 8-OH-DPAT. The mechanisms thus appear complicated, and effects of 8-OH-DPAT on post-synaptic 5HT is possibly involved. ${ }^{5}$

Biological or environmental interferences during the developmental process have been proved important in determin- 
ing the sensorimotor gating function. For example, a neonatal 5-HT depletion by pharmacological intervention with 5,7-DHT strengthens the ASR, but leaves the PPI unaffected.? However in a non-pharmacological approach, rats reared in social isolation since weanling characteristically exhibit a higher ASR and a lower PPI. ${ }^{8}$ The isolation rearing (IR)-induced gating deficit is developmentally specific, as it is unable to be ameliorated by re-socialization after so called "critical period" of early life. ${ }^{9}$ IR paradigm is thus appropriate in exploring the mechanism underlying the 5-HT1A modulation of sensorimotor gating function, in particular social interaction is reported to affect 5 -HT1A profiles. ${ }^{10}$

The interactive effects between IR and 5-HT1A receptors appear inconsistent in rodent study. IR has been reported to augment the behavioral effects of 8-OH-DPAT in rats, possibly via a mechanism of supersensitivity of the post-synaptic 5-HT1A receptor. ${ }^{11}$ On the other hand, 5-HT1A activation may reverse IR-induced PPI deficit without any effect on the ASR in mice, via a possible mechanism of presynaptic 5-HT1A autoreceptors. ${ }^{12}$ It is worth investigating whether 5-HT depletion model, as it downgrades the presynaptic 5-HT effects, could be used to explore the role of 5-HT1A receptor in the IRimpaired gating function.

The present study examined the effects of 5HT1A activation on ASR and PPI in isolation reared rats following 5-HT depletion by 5,7-DHT. The results may be helpful for a better understanding in terms of the functional role of 5-HT1A receptor in developmentally social attachment.

\section{METHODS}

\section{Animals}

Male Sprague-Dawley (SD) rats aged at 21-23 days old (weaned; BioLASCO Taiwan Co., Ltd., Yilan, Taiwan) were used in the study and were divided randomly into 2 rearing conditions [social rearing (SOC, 2 rats per cage) vs. isolation rearing (IR, housed singly)]. The animals were kept in a temperature $-\left(22 \pm 4^{\circ} \mathrm{C}\right)$ and humidity-controlled $(50 \pm 20 \%)$ room under a 12-h light/dark cycle (lights on from 07:00 to 19:00) and given ad libitum access to a standard laboratory chow diet (Ralston Purina, St. Louis, MO, USA) and sterile water. At their age of postnatal days $77-79,{ }^{13}$ rats in each rearing condition were randomly assigned to one of the two managements (sham control vs. 5-HT depletion surgery), thus made it totally 4 groups (SOC-sham, IR-sham, SOC-depletion, IR-depletion, $\mathrm{N}=8$ for each) to enter the behavioral protocol (7 days after the surgery) for measuring their ASR and PPI under different doses of 8-OH-DPAT. Locomotor activity was also monitored in both SOC and IR rats under 5-HT depleted condition. At the end of study, animals were sacrificed for an- alyzing their tissue levels of 5-HT. The study was approved by the Institutional Animal Care and Use Committee of National Defense Medical Center with the certificate number NDMC10319.

\section{Locomotor activity}

Total travel distance was used to reflect the locomotor activity, and was measured using a computerized automated activity monitoring system (MED Associates, Inc., St. Albans, VT, USA). The system included four plexiglass chambers (43 $\times 43 \times 30 \mathrm{~cm}$ ) equipped with an infrared array of 16 photodetectors and corresponding light sources that emitted photobeams $3 \mathrm{~cm}$ apart and $4.5 \mathrm{~cm}$ above the chamber floor. Travel distance was recorded at the assigned intervals and analyzed with Med-Associates software (MED Associates, Inc., St. Albans, VT, USA). Locomotor activity data were individually recorded every $5 \mathrm{~min}$ and expressed as cumulative 60-min data. ${ }^{14}$

\section{PPI testing}

The PPI experiments were carried out in four startle chambers (SR-LAB, San Diego Instrument, San Diego, CA, USA), each with a plexiglass cylinder ( $9 \mathrm{~cm}$ diameter). A speaker mounted $24 \mathrm{~cm}$ above the cylinder provided the background noise, prepulse stimuli and startle stimuli, which were controlled with the SR-LAB program. Startle responses were transduced by a piezoelectric accelerometer mounted below the cylinder, digitized and rectified. The movement of the rats was measured for $100 \mathrm{~ms}$ after startle stimulus onset. The rats were allowed to habituate to the background noise of $65 \mathrm{~dB}$ for $5 \mathrm{~min}$ after being placed into the chambers. The acoustic stimuli were calibrated every week using a digital sound level meter (RadioShack, Fort Worth, TX, USA) that was positioned inside the cylinder on the startle platform. Sixty trials were conducted in each test session, with an average inter-trial interval of $15 \mathrm{~s}$. The first and last 6 trials (in Block 1 and Block 3, respectively) each consisted of a single $40 \mathrm{~ms} 120 \mathrm{~dB}$ startle stimulus (i.e., startle stimulus alone). The middle 48 trials (Block 2) consisted of random delivery of 12 trials of startle stimulus alone, 12 no-stimulus trials, and 24 prepulse trials (i.e., prepulse stimulus with startle stimulus). The prepulse trials consisted of a single $120 \mathrm{~dB}$ startle stimulus preceded by a $20 \mathrm{~ms}$ non-startling prepulse stimulus of $12 \mathrm{~dB}$ above the background noise. The prepulse stimulus preceded the startle stimulus by $100 \mathrm{~ms}$. The startle amplitude (measured in arbitrary units) of the first 6 trials (i.e., startle stimulus alone trials). The PPI was determined with the data from Block 2 according to the following formula: [1-(startle amplitude following prepulse+pulse pair/startle amplitude following startle stimulus alone) $] \times 100 \%$. $^{15}$ 


\section{5-HT tissue levels in cortex, striatum, and hippocampus}

Rats were sacrificed by decapitation and their brains were rapidly removed. Various brain areas, including cortex, striatum, and hippocampus were rapidly dissected on an icy cold plate, then weighed and stored at $-80^{\circ} \mathrm{C}$ until homogenization with ultrasonication in $0.2 \mathrm{~mL}$ of $7 \mathrm{~N}$ perchloric acid (Sigma Chemical Industries, Ltd., Saint Louis, MO, USA). Homogenates were centrifuged at $12,000 \times \mathrm{g}$ for $30 \mathrm{~min}$ at $4^{\circ} \mathrm{C}$.

The concentration of serotonin (5-HT) was determined by high performance liquid chromatography (HPLC) equipped with electrochemical detector (ECD, LC-4C, BAS, West Lafayette, IN, USA) (10 nA, filter $2.0 \mathrm{~Hz}$, AppE cell $0.750 \mathrm{~V})$, autosampler (Shimadzu SIL-10ADvp autosampler, Shimadzu, Japan). The supernatant was filtered through a 0.22 um filter, and was analyzed by HPLC equipped with an Alltima ${ }^{\mathrm{TM}}$ (Grace Davison Discovery Scienes, Ltd., Deerfield, IL, USA) reversedphase $\mathrm{C} 18$ column $(4.6 \times 150 \mathrm{~mm}, 5 \mu \mathrm{m})$. Injection volume was $20 \mathrm{uL}$. The mobile phase contains $100 \mathrm{mM} \mathrm{NaH}{ }_{2} \mathrm{PO}_{4} \cdot \mathrm{H}_{2} \mathrm{O}$, $0.74 \mathrm{mM}$ sodium octanesulfonate (SOS), $0.02 \mathrm{mM}$ EDTA and $20 \%$ methanol adjusted the $\mathrm{pH}$ to 3.0 by $\mathrm{H}_{3} \mathrm{PO}_{4}$ and with a flow rate of $1.0 \mathrm{~mL} / \mathrm{min}$. The calibration curve was obtained based on 6 levels $(20,40,60,80,100$, and 200 ppm) of 5-HT standard (Sigma Chemical Industries, Ltd.). The coefficient of determination $\left(\mathrm{r}^{2}\right)$ was greater than 0.995 .

\section{Surgery}

Microinjection needle (Hamilton, 30-gauge, $10 \mu \mathrm{L}$ ) was used to inject 5,7-DHT into ICV (AP: $-0.8 \mathrm{~mm}$ from bregma, L: $\pm 1.5 \mathrm{~mm}$ from the midline, DV: $-0.4 \mathrm{~mm}$ from dura $)^{16}$ with a flow rate of $1 \mu \mathrm{L} / \mathrm{min}$ controlled by a microinjection pump (CMA/100, Carnegie, Medicin, Sweden) for $10 \mathrm{~min}$.

\section{Drugs}

Serotoninergic neurotoxin 5,7-DHT (200 $\mu$ g, Sigma-Aldrich, St. Louis, MO, USA) was dissolved in saline solution with $0.6 \%$ ascorbic acid and was administered via intracerebraoventricular (ICV) injection. $30 \mathrm{~min}$ before the 5,7-DHT injection, desipramine ( $25 \mathrm{mg} / \mathrm{kg}$, Sigma-Aldrich), a reuptake inhibitor of norepinephrine, was administered intraperitoneally to protect noradrenergic neurons from neurotoxicity. ${ }^{17}$ To activate the 5 -HT1A receptors, 8 -OH-DPAT (Sigma-Aldrich) was intraperitoneally injected (0, 10, and 100 $\mu \mathrm{g} / \mathrm{kg}$, in a volume of $1 \mathrm{~mL} / \mathrm{kg}$ ) $10 \mathrm{~min}$ prior to the behavioral testing.

\section{Data analyses}

Statistical analyses were performed across the groups via a multi-factor analysis of variance (ANOVA) by using SPSS Version 18.0 (SPSS Inc., Chicago, IL, USA), with REARING
CONDITION and LESION as between-subject factors. Further analyses with post-hoc multiple comparisons were performed where possible. All data were expressed as mean \pm standard error of the mean (SEM). A p value of $<0.05$ was considered statistically significant.

\section{RESULTS}

\section{Effects of 5,7-DHT on tissue 5-HT levels}

The HPLC method confirmed the reductions of tissue 5-HT caused by 5,7-DHT in cortex, striatum, and hippocampus in which IR rats were less affected (for SOC rats, cortex: $32 \%$, striatum: $35 \%$, hippocampus: $33 \%$; for IR rats, cortex: $73 \%$, striatum: $51 \%$, hippocampus: $61 \%$ ) (Table 1). There were main effects of LESION $[F(1,16)=9.61, p<0.01]$ and REARING CONDITION $[\mathrm{F}(1,16)=5.06, \mathrm{p}<0.05]$ in striatum, and main effects of LESION $[\mathrm{F}(1,16)=9.40, \mathrm{p}<0.01]$ and REARING CONDITION $[\mathrm{F}(1,16)=5.24, \mathrm{p}<0.05]$ in hippocampus with REARING CONDITION $\times$ LESION interaction $[\mathrm{F}(1,16)=$ $4.88, \mathrm{p}<0.05]$. For cortex, there was a main effect of LESION $[\mathrm{F}(1,16)=6.15, \mathrm{p}<0.05]$.

\section{Effects of 5,7-DHT on locomotor activity}

IR rats exhibited greater locomotor activity (indexed by total travel distance) in the 60 min activity test $[\mathrm{t}(30)=3.4, \mathrm{p}<$ 0.01 ]. The treatment of 5,7-DHT did not cause any effect on locomorot activity in rats of both rearing conditions [no effects of LESION or REARING CONDITION $\times$ LESION; main effect of REARING CONDITION remained, $\mathrm{F}(1,28)=$ 9.02, $\mathrm{p}<0.01$ ] (Figure 1).

\section{Effects of 5,7-DHT on ASR and PPI}

Both REARING CONDITION and LESION have no effects on the ASR. For PPI, IR rats was inferior than SOC rats [main effects of REARING CONDITION, F(1,28)=7.98,

Table 1. 5-HT concentration (pg/mg) in cortex, striatum, and hippocampus ( $\mathrm{N}=5$ for each group)

\begin{tabular}{lcc}
\hline \multicolumn{1}{c}{ Region } & 5-HT sham & 5-HT lesion \\
\hline SOC rats & & \\
Cortex & $896.3 \pm 412.5$ & $289.9 \pm 60.8^{*}$ \\
Striatum & $1136.7 \pm 99.6$ & $401.1 \pm 125.0^{\dagger}$ \\
$\quad$ Hippocampus & $1135.3 \pm 404.3$ & $377.6 \pm 80.8^{\ddagger}$ \\
IR rats & & \\
Cortex & $335.4 \pm 129.3$ & $247.9 \pm 38.2^{\S}$ \\
Striatum & $484.9 \pm 169.3$ & $245.5 \pm 58.6$ \\
Hippocampus & $558.5 \pm 187.5$ & $343.2 \pm 61.4$ \\
\hline
\end{tabular}

Comparisons between sham and lesion groups (all $\mathrm{p}<0.05) .{ }^{*} \mathrm{t}(8)=$ $2.41,{ }^{t} \mathrm{t}(8)=2.68,{ }_{\mathrm{t}}(8)=2.09, \mathrm{~s} \mathrm{t}(8)=1.90 .5-\mathrm{HT}$ : serotonin, SOC: social, IR: isolation-rearing 
$\mathrm{p}<0.01]$. The treatment of 5,7-DHT did not cause any effect on PPI in rats of both rearing conditions [no effects of LESION or REARING CONDITION×LESION] (Figure 2).

\section{Effects of 8-OH-DPAT on ASR and PPI in 5-HT depleted rats reared in social isolation}

For ASR, 8-OH-DPAT has no effect on the ASR in SOC rats. However, it strengthens the ASR in both IR-sham and IR-depletion rats [for IR rats, main effect of DOSE, $F(2,28)=6.11$, $\mathrm{p}<0.01$ ] (Figure 3).

For PPI, LESION was a key factor determining the 8-OHDPAT effect, as the drug at $100 \mu \mathrm{g} / \mathrm{kg}$ caused a higher PPI in 5-HT depleted SOC rats than their sham controls [for SOC rats, main effect of LESION, $\mathrm{F}(1,14)=4.90, \mathrm{p}<0.05$ and effect of LESION $\times$ DOSE $F(2,28)=3.73, p<0.05]$. However, this effect was reversed in an opposite manner for IR rats, in which the 8-OH-DPAT at $100 \mu \mathrm{g} / \mathrm{kg}$ strengthens the PPI in non-lesioned IR rats but downgrades the PPI in lesioned IR rats [for
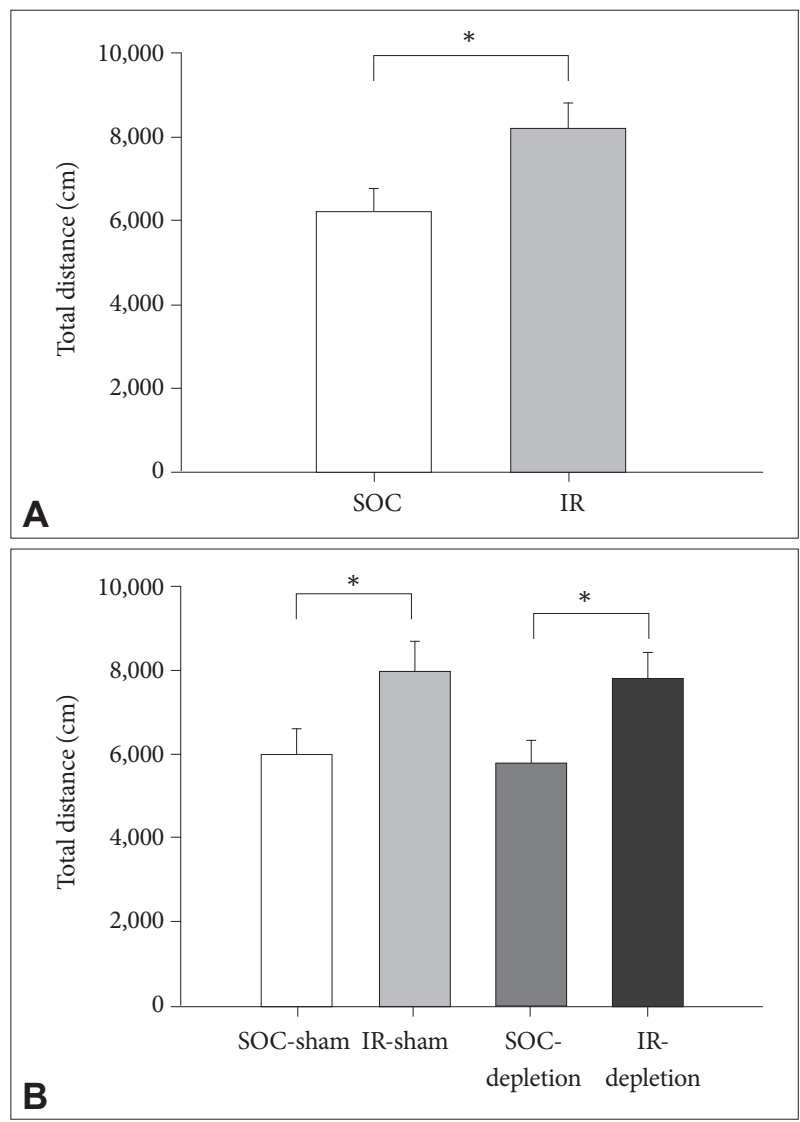

Figure 1. Effects of 5-HT depletor, 5,7-DHT on locomotor activity in isolation reared rats after $5 \mathrm{HT}$ depletion. A: IR rats are hyperactive than social controls. B: 5,7-DHT has no effects on locomotor activity in rats of both rearing conditions. Data are represented by mean \pm SEM. ${ }^{*} p<0.01, N=16$ for $S O C$ and IR rats $(A)$ and $N=8$ for rats under different rearing and treatment conditions (B). 5-HT: serotonin, SOC: social, IR: isolation-rearing.
IR rats, main effect of lesion, $\mathrm{F}(1,14)=9.38, \mathrm{p}<0.01$ and effect of lesion $\times$ dose $F(2,28)=5.52, \mathrm{p}<0.01$ ] (Figure 4).

\section{DISCUSSION}

The major finding of our study is that 5-HT depletion may lead to distinctive 8-OH-DPAT effects on PPI in rats, depending on their rearing conditions. In the present study, $8-\mathrm{OH}$ DPAT at $100 \mu \mathrm{g} / \mathrm{kg}$ improved PPI in 5-HT depleted SOC rats, whereas it downgraded PPI in the 5-HT depleted IR rats. In other words, the integrity of 5-HT neuron is important for the 5-HT1A modulatory effects on sensorimotor gating function, in which the development of social attachment may serve as a key factor in determining the effects.

Locomotor hyperactivity and impairment of PPI are two prominent characteristics in rodent model of IR, and were firstly validated in the present study. Rather than hyperactivi-

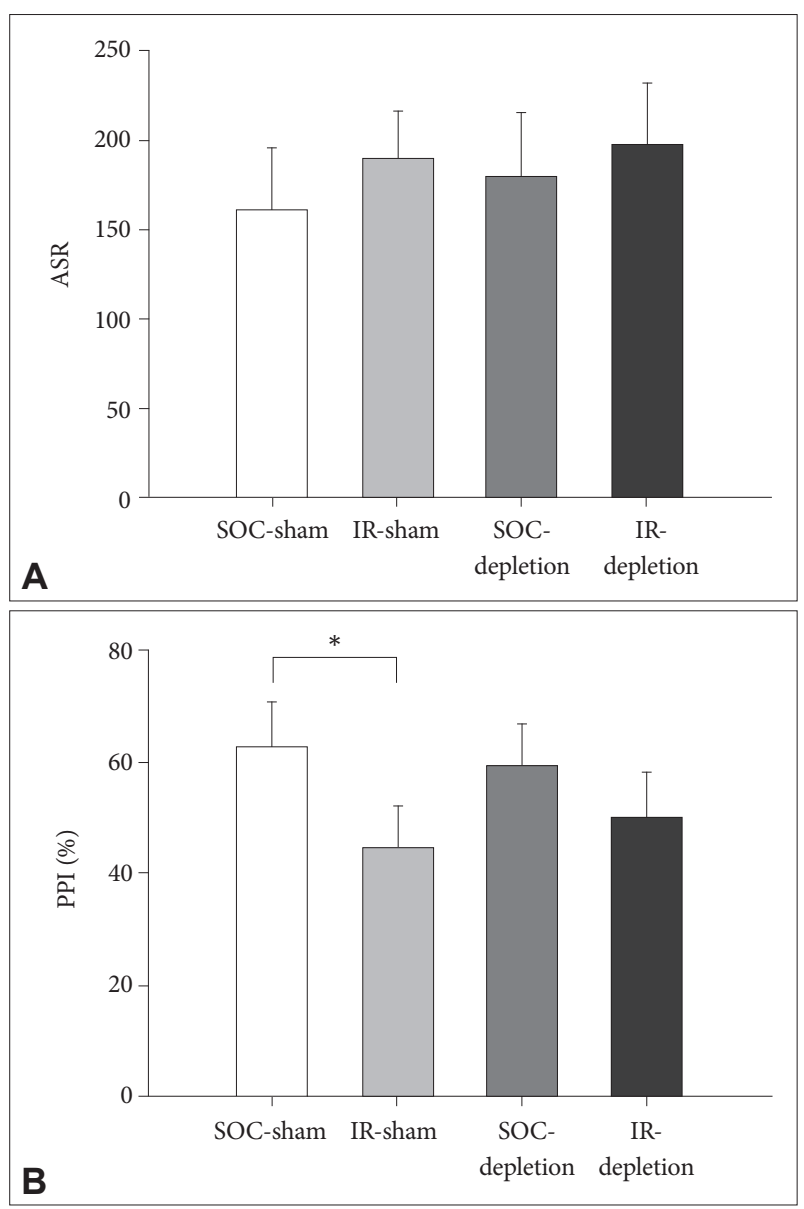

Figure 2. Effects of 5-HT depletor, 5,7-DHT on ASR and PPI in isolation reared rats after $5 \mathrm{HT}$ depletion. A: Both REARING CONDITION and LESION have no effects on the ASR. B: IR rats perform lower PPI than social controls; 5,7-DHT has no effects on PPI in rats of both rearing conditions. Data are represented by mean \pm SEM. * $\mathrm{p}<0.01, \mathrm{~N}=8$ for each group. $5-\mathrm{HT}$ : serotonin, SOC: social, IR: isolation-rearing, ASR: acoustic startle response, PPI: prepulse inhibition. 
ty, PPI is more appropriate in addressing developmental specificity because the IR-induced PPI deficit is unable to be reversed by resocialization later. ${ }^{9}$ The present study demonstrated that the depletion of central 5-HT did not modulate the IR effects on both locomotor activity and sensorimotor gating
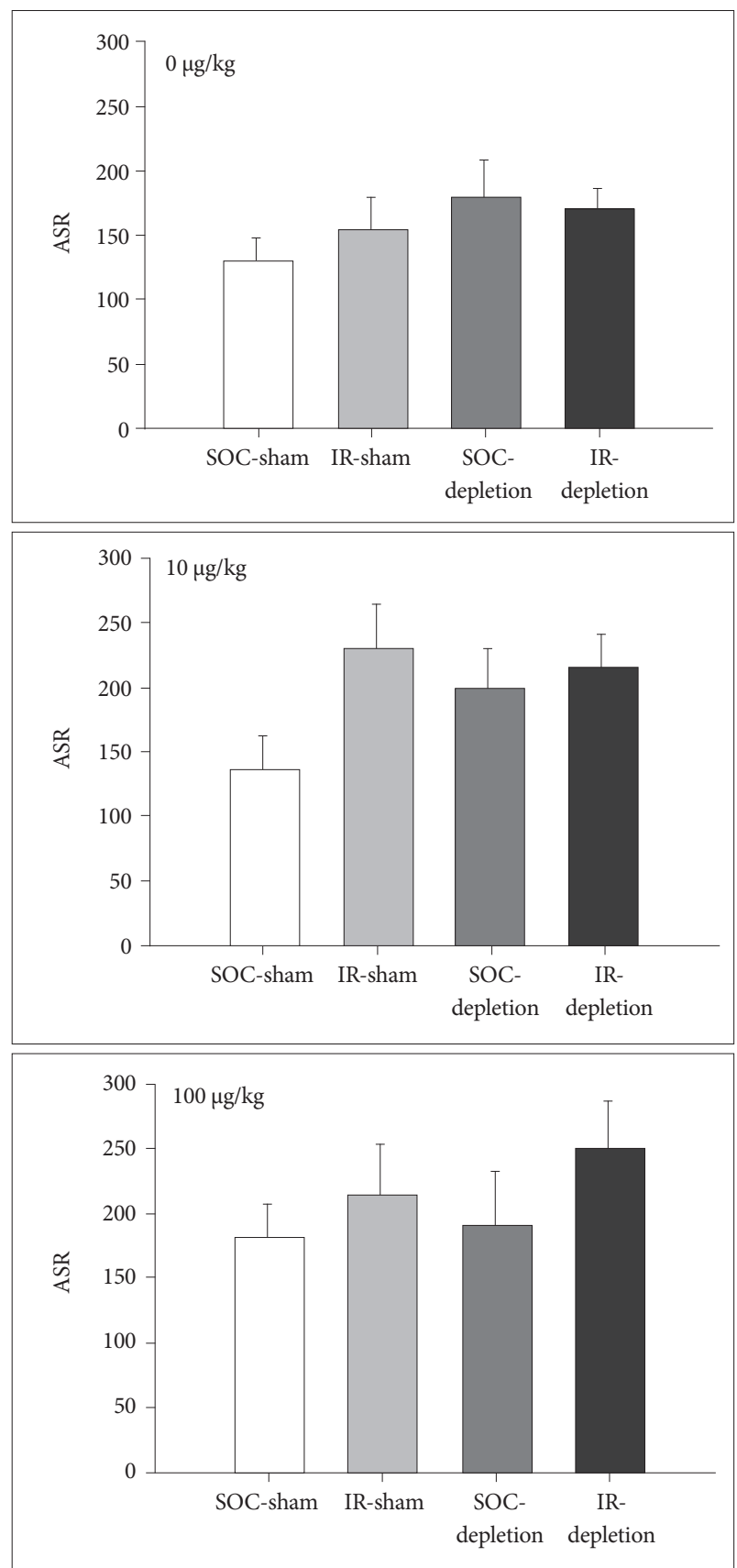

Figure 3. Effects of different doses of 8-OH-DPAT on ASR in isolation reared rats after $5 \mathrm{HT}$ depletion. A: 8-OH-DPAT has no effect on the ASR in SOC rats (comparisons were made among upper, middle and bottom panels). B: 8-OH-DPAT strengthens the ASR in both IR-sham and IR-depletion rats (comparisons were made among upper, middle and bottom panels). Data are represented by mean \pm SEM, $\mathrm{N}=8$ for each group. $5-\mathrm{HT}$ : serotonin, SOC: social, IR: isolation-rearing, ASR: acoustic startle response. function (including ASR and PPI). Note the condition of 5-HT depletion leads to a damage to central 5-HT-containing axons and terminals, ${ }^{18}$ however the 5-HT efflux appeared unaffected in lesioned rats compared to the sham control rats, in both the baseline and the anxiety triggered conditions. ${ }^{19,20} \mathrm{~A}$ compensatory mechanism is therefore highly suggested for the maintenance of synaptic function. ${ }^{21}$ Our data implies that this mechanism is preserved in rats reared in social isolation.
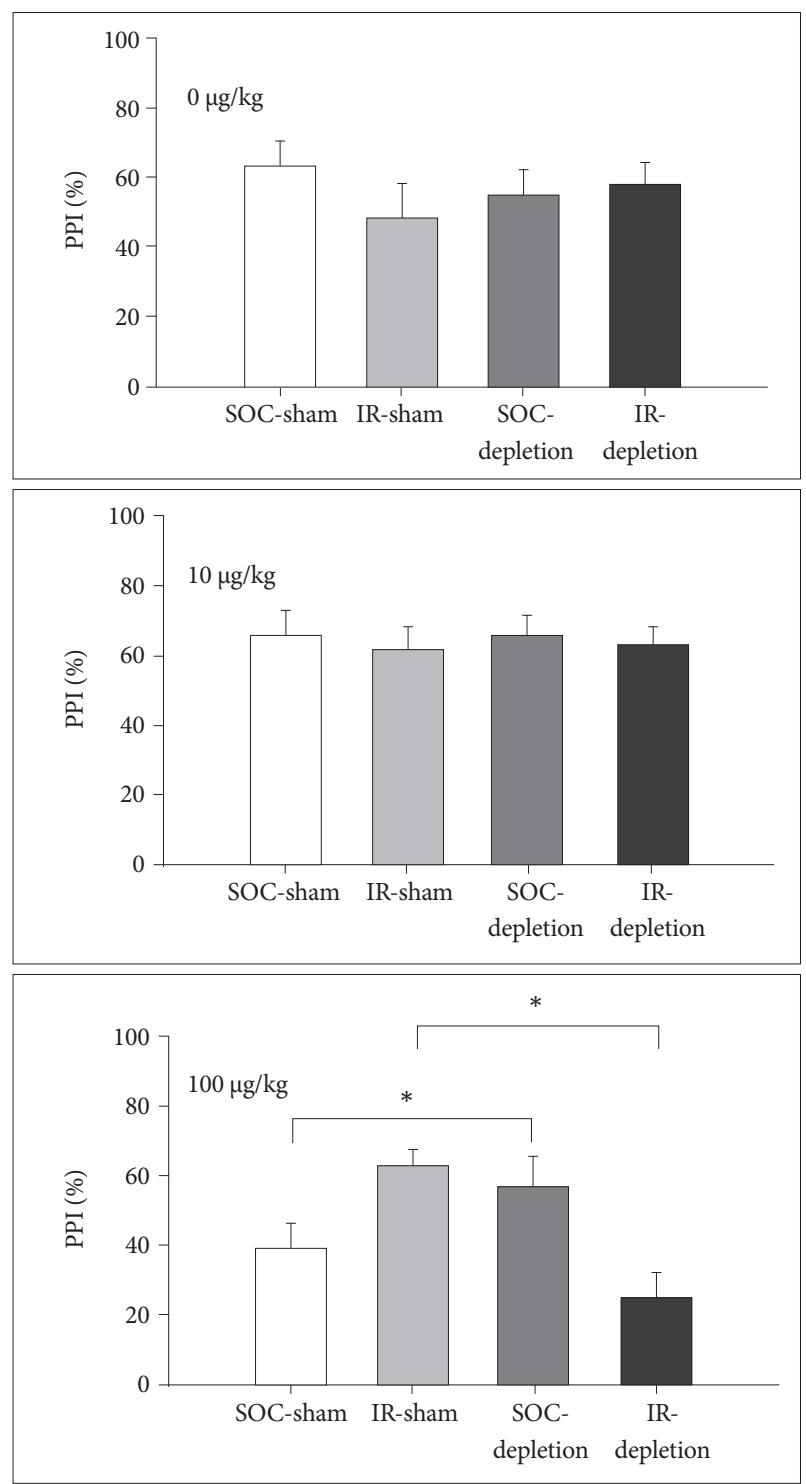

Figure 4. Effects of different doses of 8-OH-DPAT on PPI in isolation reared rats after $5 \mathrm{HT}$ depletion. A: LESION was a key factor determining the 8-OH-DPAT effect, as the drug at higher dose (i.e., $100 \mu \mathrm{g} / \mathrm{kg}$ ) caused a higher PPI in 5-HT depleted SOC rats than their sham controls (comparisons were made among upper, middle and bottom panels). B: 8-OH-DPAT strengthens the PPI in nonlesioned IR rats. For lesioned IR rats, higher dose $(100 \mu \mathrm{g} / \mathrm{kg}) 8-\mathrm{H}-$ DPAT downgrades the PPI (comparisons were made among upper, middle and bottom panels). Data are represented by mean \pm SEM. ${ }^{*} \mathrm{p}<0.01, \mathrm{~N}=8$ for each group. $5-\mathrm{HT}$ : serotonin, SOC: social, IR: isolation-rearing, PPI: prepulse inhibition. 
The most striking finding of the present study is the effect of 8-OH-DPT on sensorimotor gating function in IR rats, particularly in the lesioned condition. For ASR, 8-OH-DPT boosted it in IR but not SOC rats. 8-OH-DPT was previously reported to increase the amplitude of startle in a similar acoustic paradigm..$^{22}$ Since in the present study the 8-OH-DPT-increased ASR displayed in both the sham control and the 5,7DHT pretreated rats, the effect should be less relevant to the lesion condition of 5-HT neurons. In other words, the nature of hyper-reactivity to startle stimulus of IR rats ${ }^{8,23}$ is presumably more relevant to 5-HT1A activation than the integrity of 5-HT neurons.

In contrast to ASR, the IR-induced impairment of PPI represents a failure of information-filtering to mitigate the response to the ensuing stimuli (i.e., a dysfunction of the central inhibitory ability), ${ }^{24}$ and the integrity of 5-HT neurons rather than rearing condition became the key to affect the 8-OH-DPT effects on PPI. High dose of 8-OH-DPT (at $100 \mu \mathrm{g} / \mathrm{kg}$ ) caused a greater PPI in 5-HT depleted SOC rats than their sham controls, indicating that 5-HT1A activation-downgraded $\mathrm{PPI}^{2,3}$ can be modulated if 5-HT neurons are not intact, possibly via a compensatory mechanism as mentioned above. ${ }^{21}$ However, the mechanism appears different in the IR rats, as in the present study the 8-OH-DPT strengthened the PPI in IR-sham rats but downgraded it in IR-lesioned rats. In other word, processes responsible for maintaining the function of synaptic 5-HT transmission of IR rats in the condition of 5,7-DHT impact have altered now due to the activation of 5-HT1A receptors.

The mechanisms underlying these observations might be complicated, however several points are worth addressing. First, it implies that the two pharmacological managements (5,7-DHT and 8-OH-DPT) exert their effects on sensorimotor gating in IR rats via different pathways. While 5,7-DHT exerts its effects presynaptically, 8-OH-DPT executes it effects pre and post-synaptically, because 5-HT1A receptors may be expressed in both places. ${ }^{25,26}$ Second, 8-OH-DPT at the dose used in the present study (i.e., $100 \mu \mathrm{g} / \mathrm{kg}$ ) is generally inadequate to activate the postsynaptic 5-HT1A receptors, ${ }^{27}$ however since the IR rats characteristically may have a supersensitive profile of the post-synaptic 5-HT1A receptor, ${ }^{11}$ the 5,7DHT- readjusted presynaptic function may affect the tonicity/ balance between pre and post-synaptic 5-HT1A receptors, and therefore responsible for the diverse effects of 8-OH-DPT on PPI in IR-sham and IR-depletion rats. Finally, although 8-OHDPAT is a potent agonist at 5-HT1A receptors, it may also antagonize the effects of 5-HT itself, suggesting an effect of partial agonism, ${ }^{28}$ which is along with the dose-dependent, biphasic effects of 8-OH-DPAT. ${ }^{29}$ This may be relevant also to the diverse effects of 8-OH-DPAT on PPI in IR-sham and IRdepletion rats.
Finally, as IR downgraded the tissue concentration of 5HT in our data and others, ${ }^{30}$ IR rats, to a degree, appear equivalent to the 5-HT depleted SOC rats in terms of 5-HT-related performance, and thus the impact of 5,7-DHT in IR rats accordingly becomes less than that of SOC rats (Table 1). This hypothesis, together with an enhanced postsynaptic 5- HT1A activity of IR, ${ }^{5,11}$ may partially explain the observation that the effects of 8-OH-DAPT on PPI in SOC-depletion rats were similar to that of IR-sham IR rats.

In conclusion, the present study demonstrated a paradigm of using 5-HT depletion model in exploring the role of 5-HT1A receptor in the gating dysfunction induced by a lack of social connection since early life. The integrity of central 5-HT system is a key factor to modulate the sensorimotor gating only when the 5-HT1A is activated, in which the post-weaning social attachment is highly pertinent to the modulatory outcome.

\section{Acknowledgments}

This research was supported by grants from the National Defense Medical Center, Taiwan (MAB101-85 and MAB102-83), Ministry of Science and Technology (NSC 96-2314-B-073-001, MOST 105-2410-H-016-002MY2), and the Beitou branch of Tri-Service General Hospital (TSGHBT-104-02, TSGH-BT-105-003).

\section{REFERENCES}

1. Nanry KP, Tilson HA. The role of 5HT1A receptors in the modulation of the acoustic startle reflex in rats. Psychopharmacology (Berl) 1989;97: 507-513.

2. Rigdon GC, Weatherspoon JK. 5-Hydroxytryptamine 1a receptor agonists block prepulse inhibition of acoustic startle reflex. J Pharmacol Exp Ther 1992;263:486-493.

3. Sipes TA, Geyer MA. 8-OH-DPAT disruption of prepulse inhibition in rats: reversal with (+)WAY 100,135 and localization of site of action. Psychopharmacology (Berl) 1995;117:41-48.

4. Müller CP, Carey RJ, Huston JP, De Souza Silva MA. Serotonin and psychostimulant addiction: focus on 5-HT1A-receptors. Prog Neurobiol 2007;81:133-178.

5. Fletcher PJ, Selhi ZF, Azampanah A, Sills TL. Reduced brain serotonin activity disrupts prepulse inhibition of the acoustic startle reflex. Effects of 5,7-dihydroxytryptamine and p-chlorophenylalanine. Neuropsychopharmacology 2001;24:399-409.

6. Kusljic S, Copolov DL, van den Buuse M. Differential role of serotonergic projections arising from the dorsal and median raphe nuclei in locomotor hyperactivity and prepulse inhibition. Neuropsychopharmacology 2003;28:2138-2147.

7. Kołomańska P, Wyszogrodzka E, Rok-Bujko P, Krząścik P, Kostowski W, Zaniewska M, et al. Neonatal serotonin (5-HT) depletion does not disrupt prepulse inhibition of the startle response in rats. Pharmacol Rep 2011;63:1077-1084.

8. Wilkinson LS, Killcross SS, Humby T, Hall FS, Geyer MA, Robbins TW. Social isolation in the rat produces developmentally specific deficits in prepulse inhibition of the acoustic startle response without disrupting latent inhibition. Neuropsychopharmacology 1994;10:61-72.

9. Liu YP, Kao YC, Tung CS. Critical period exists in the effects of isolation rearing on sensorimotor gating function but not locomotor activity in rat. Prog Neuropsychopharmacol Biol Psychiatry 2011;35:1068-1073.

10. Rasmuson S, Olsson T, Henriksson BG, Kelly PA, Holmes MC, Seckl JR, et al. Environmental enrichment selectively increases 5-HT1A re- 
ceptor mRNA expression and binding in the rat hippocampus. Brain Res Mol Brain Res 1998;53:285-290.

11. Wright IK, Ismail H, Upton N, Marsden CA. Effect of isolation rearing on 5-HT agonist-induced responses in the rat. Psychopharmacology (Berl) 1991;105:259-263.

12. Sakaue M, Ago Y, Baba A, Matsuda T. The 5-HT1A receptor agonist MKC-242 reverses isolation rearing-induced deficits of prepulse inhibition in mice. Psychopharmacology (Berl) 2003;170:73-79.

13. Yang YY, Lu CL, Lo SM, Peng CH, Liu YP. Early antipsychotic intervention and schizophrenia. Med Hypotheses 2015;85:367-370.

14. Ko CY, Liu YP. Isolation rearing impaired sensorimotor gating but increased pro-inflammatory cytokines and disrupted metabolic parameters in both sexes of rats. Psychoneuroendocrinology 2015;55:173-183.

15. Ko CY, Liu YP. Disruptions of sensorimotor gating, cytokines, glycemia, monoamines, and genes in both sexes of rats reared in social isolation can be ameliorated by oral chronic quetiapine administration. Brain Behav Immun 2016;51:119-130.

16. Paxinos G, Watson C. The Rat Brain in Stereotaxic Coordinates (6th Ed.). New Tork: Academic Press; 2008.

17. Wang JY, Shum AY, Lin TC, Wang Y. Central serotonergic lesions increase voluntary alcohol consumption in Sprague Dawley rats: moderation by long-term ethanol administration. Alcohol Clin Exp Res 1996; 20:1252-1259.

18. Baumgarten HG, Björklund A. Neurotoxic indoleamines and monoamine neurons. Annu Rev Pharmacol Toxicol 1976;16:101-111.

19. Kalén P, Strecker RE, Rosengren E, Björklund A. Endogenous release of neuronal serotonin and 5-hydroxyindoleacetic acid in the caudate-putamen of the rat as revealed by intracerebral dialysis coupled to high-performance liquid chromatography with fluorimetric detection. J Neurochem 1988;51:1422-1435.

20. Rex A, Thomas H, Hörtnagl H, Voits M, Fink H. Behavioural and microdialysis study after neurotoxic lesion of the dorsal raphe nucleus in rats. Pharmacol Biochem Behav 2003;74:587-593.
21. Kirby LG, Kreiss DS, Singh A, Lucki I. Effect of destruction of serotonin neurons on basal and fenfluramine-induced serotonin release in striatum. Synapse 1995;20:99-105.

22. Conti LH. Interactions between corticotropin-releasing factor and the serotonin $1 \mathrm{~A}$ receptor system on acoustic startle amplitude and prepulse inhibition of the startle response in two rat strains. Neuropharmacology 2012;62:256-263.

23. Bakshi VP, Swerdlow NR, Braff DL, Geyer MA. Reversal of isolation rearinginduced deficits in prepulse inhibition by Seroquel and olanzapine. Biol Psychiatry 1998;43:436-445.

24. Swerdlow NR, Braff DL, Geyer MA. Animal models of deficient sensorimotor gating: what we know, what we think we know, and what we hope to know soon. Behav Pharmacol 2000;11:185-204.

25. Hall MD, Mestikawy SE, Emerit MB, Pichat L, Hamon M, Gozlan H. [3H]8-Hydroxy-2-(di-n-propylamino)tetralin binding to pre- and postsynaptic 5-hydroxytryptamine sites in various regions of the rat brain. J Neurochem 1985;44:1685-1696.

26. Azmitia EC, Gannon PJ, Kheck NM, Whitaker-Azmitia PM. Cellular localization of the 5-HT1A receptor in primate brain neurons and glial cells. Neuropsychopharmacology 1996;14:35-46.

27. Rex A, Fink H. Effects of 8-OH-DPAT on hippocampal NADH fluorescence in vivo in anaesthetized rats. J Neurosci Res 2006;83:551-556.

28. Hadrava V, Blier P, de Montigny C. Partial agonistic activity of R- and S-enantiomers of 8-OH-DPAT at 5-HT1A receptors. J Psychiatry Neurosci 1996;21:101-108.

29. Harrison AA, Markou A. Serotonergic manipulations both potentiate and reduce brain stimulation reward in rats: involvement of serotonin1A receptors. J Pharmacol Exp Ther 2001;297:316-325.

30. Brenes JC, Rodríguez O, Fornaguera J. Differential effect of environment enrichment and social isolation on depressive-like behavior, spontaneous activity and serotonin and norepinephrine concentration in prefrontal cortex and ventral striatum. Pharmacol Biochem Behav 2008;89:85-93. 